\title{
Prospective evaluation of biliary reconstruction with duct-to-duct continuous suture in adult live donor liver transplantation
}

\author{
Tung Yu Tsui • Hans Jürgen Schlitt • Aiman Obed
}

Received: 11 February 2010 /Accepted: 10 June 2010 /Published online: 8 November 2010

(C) The Author(s) 2010. This article is published with open access at Springerlink.com

\begin{abstract}
Purpose Biliary reconstruction remains the Achilles' heel of adult live donor liver transplantation (LDLT). The study aims to investigate the feasibility of duct-to-duct hepaticocholedochostomy in LDLT.

Methods Perioperative data from 30 consecutive LDLT aiming at duct-to-duct reconstruction of the biliary tract using a continuous suture technique were prospectively collected. Nineteen recipients $(63.3 \%)$ had one graft bile duct. Eleven recipients $(36.7 \%)$ had two or three graft bile ducts. The median follow-up was 50 months.

Results The overall biliary complication rate was $23.3 \%$. Two recipients developed biliary stricture $(6.7 \%)$, and two recipients $(6.7 \%)$ presented with biliary leakage in early posttransplant phase ( $<90$ days). One recipient suffered from bilioma (3.3\%), and two recipients $(6.7 \%)$ presented with biliary stricture in later posttransplant phase ( $>90$ days). No correlation was found between the number of graft bile ducts and the incidence of biliary complications. No biliary
\end{abstract}

Hans Jürgen Schlitt and Aiman Obed share the senior authorship of this work.

T. Y. Tsui $\cdot$ H. J. Schlitt $\cdot$ A. Obed

Department of Surgery, University of Regensburg Medical Center,

Franz-Josef-Strauss-Allee 11,

93053 Regensburg, Germany

Present Address:

T. Y. Tsui $(\bowtie)$

Department of Hepatobiliary and Transplant Surgery,

University Medical Center Hamburg-Eppendorf,

20246 Hamburg, Germany

e-mail: tytsui@gmail.com

Present Address:

A. Obed

Department of Surgery, University Medical Center Göttingen,

Göttingen, Germany complication-associated necessity for re-transplantation or mortality was observed. On multivariate analysis, no single risk factor associated with biliary complication could be identified. All biliary complications were successfully treated with Roux-en-hepaticojejunostomy and/or with endoscopic interventions.

Conclusion Duct-to-duct hepaticocholedochostomy with continues suture represents a safe and feasible procedure for biliary reconstruction in LDLT. Recipients may benefit from aggressive management of biliary complications with Roux-en-hepaticojejunostomy as compared with repeated endoscopic interventions in early posttransplant phase.

Keywords Live donor liver transplantation - Biliary reconstruction · Duct-to-duct continues suture $\cdot$ Biliary complication

\section{Introduction}

Adult living donor liver transplantation (LDLT) has been successfully established to save the life of patients with end-staged liver diseases and to reduce the need of deceased donation [1]. The surgical techniques of LDLT have been largely improved in the past decade, reflected by the low morbidity of donor hepatectomy and high graft and recipient survivals [2]. However, the reconstruction of the biliary tract remains one of the largest challenges to liver transplant surgeons [3]. A high incidence of biliary complications has been reported, reaching up to $67 \%$, in early series of studies [3-7]. Through the technical refinement, the incidence of biliary complications has been reduced, but still reaches up to $40 \%$ in recent studies [8-10].

The high incidence of biliary complications in LDLT is mainly associated with various factors, including the 
anatomical variation of the biliary tract, the microcirculatory supply of bile duct, the arterial complications, and the experience of surgeons. Duct-to-duct anastomosis is currently a favorable method to reconstruct the biliary tract in LDLT. Technically easier manipulation and the preservation of physiologic bilioenteric continuity are two main advantages over Roux-en-Y hepaticojejunostomy by performing duct-to-duct anastomosis. However, a continuously high incidence of leakage and stricture at the anastomic site indicates that duct-to-duct anastomosis is still the Achilles' heel in LDLT. In this cohort study, we analyzed our series of LDLT with regard to decision-making in the reconstruction of the biliary tract with respect to anatomical variations and in case of biliary complications, the ways of management.

\section{Patients and methods}

Patients and study design

A cohort study aiming to analyze the feasibility of duct-toduct reconstruction of the biliary tract in LDLT was conducted. Between March 2004 and December 2006, 32 adult patients with end-staged liver diseases received right lobe liver grafts from live donors at the University of Regensburg Medical Center. Hepaticocholedochostomy was performed in 30 of 32 recipients. Two of 32 recipients received Roux-en-Y hepaticojejunostomy due to the anatomical impossibility; a combined LDLT and Whipple's operation was performed in one case, and other one was due to the presence of four bile ducts in liver graft. These two patients were, therefore, excluded from this study.

\section{Donor evaluation}

An Evaluation Program for Living Donor Liver Transplantation including $\mathrm{ABO}$ compatibility, normal liver function, and negative serology for viral hepatitis was established at the University of Regensburg Medical Center and was approved by government according to the German Transplantation Law. Computer tomography with volumetry was performed to determine the size of the right lobe graft with or without involvement of the middle hepatic vein. Approximately $1 \%$ of estimated graft weight/recipient weight was considered as a suitable ratio for live donation.

\section{Donor hepatectomy}

A right hepatectomy (liver segments $\mathrm{V}$ to VIII) was performed without vascular inflow occlusion as described. Intraoperative cholangiography via the cystic duct cannulation was routinely performed in order to define the exact anatomy of the biliary tract and to identify the line of the transection of the right hepatic duct. The right hepatic duct was divided sharply using fine scissors with at least $1-\mathrm{mm}$ margin from the bifurcation prior to parenchymal transection. The number of hepatic duct orifices was carefully re-examined in backtable. The hepatic ducts were carefully rinsed with saline before implantation.

\section{Recipient operation and reconstruction of biliary tract}

Standard LDLT was performed after total hepatectomy with preservation of the inferior vena cava as described. Following portal reperfusion and hemostasis, the hepatic artery was reconstructed via end-to-end anastomosis between donor right hepatic artery and recipient right hepatic artery (in the majority of cases) using 8-0 Prolene. Biliary continuity was achieved via hepaticocholedochostomy in 30 of 32 cases and Roux-en-Y choledochojejunostomy in two of 32 recipients.

Principally, hepaticocholedochostomy was aimed at all cases, unless more than three hepatic duct orifices were found in the liver graft. All duct-to-duct anastomosis was performed with a continuous suture using 6-0 PDS with the BV-1 fine needle. In liver grafts with one bile duct, the anastomosis was performed in end-to-end or end-to-side manner (Fig. 1a), which is dependent on the congruence of donor/recipient bile ducts. In liver grafts with two or three bile ducts, the adjacent hepatic ducts were primarily sutured together to create a common orifice followed by an end-to-end anastomosis (Fig. 1b, c). If one of the hepatic ducts located separately, an additional end-to-end anastomosis with the recipient hepatic duct, cystic duct, or end-to-side anastomosis with recipient common bile duct would be performed (Fig. 1d-f). The accessory bile ducts with diameter less than $1 \mathrm{~mm}$ were ligated. The placing of an intraductal stent or T-drainage was not routinely indicated in our series of LDLT.

\section{Postoperative management}

Immunosuppressive therapy following liver transplantation consisted of a Cyclosporin A-based drug regimen with MMF and steroids. Steroids were generally tapered within 3 months after transplantation. Postoperative ICU stay was indicated for all donors and recipients. Doppler ultrasound was performed daily for the first three postoperative days and then whenever clinically indicated. CMV prophylaxis was given to all recipients with a negative CMV serology receiving a liver graft with positive CMV serology.

Statistical analysis

All patient data were analyzed with statistical software (SPSS version 13). The differences of means between groups were compared with one-way ANOVA. $P<0.05$ was considered as statistical significance. 
a
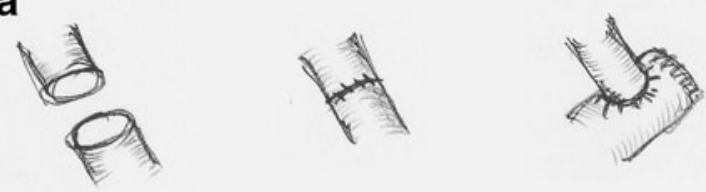

b
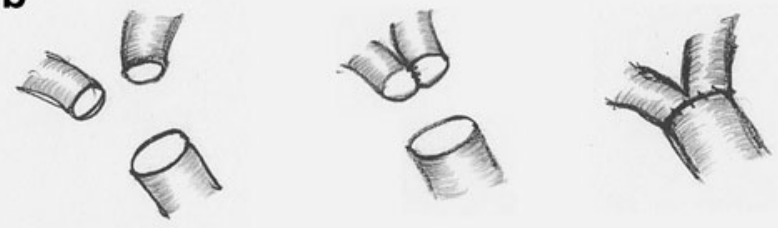

C
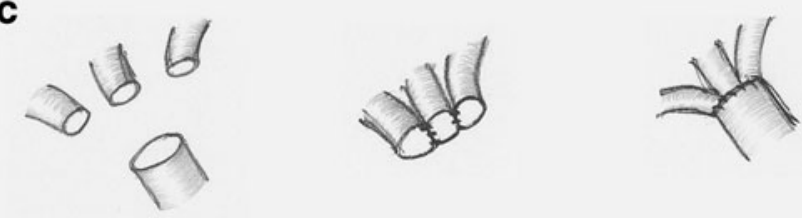

d
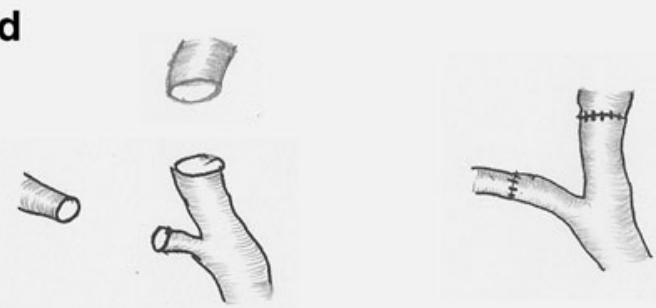

e

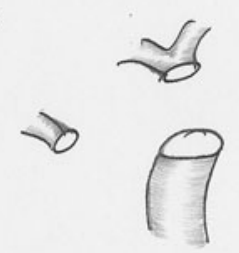

f

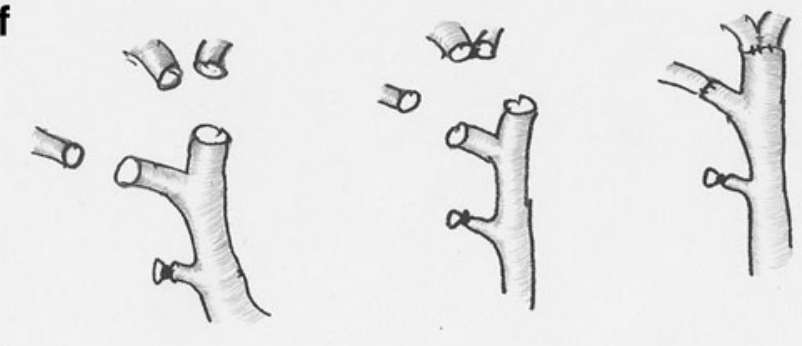

Fig. 1 Surgical techniques for bile duct reconstruction using continues suture in live donor liver transplantation. a One duct and one end-to-end or end-to-side anastomosis that based on the size of lumen. b Two ducts with one common truck and one anastomosis. c Three ducts with one common truck and one anastomosis. d Two ducts with end-to-end anastomosis to common hepatic duct and cystic duct separately. e Two ducts with end-to-side anastomosis to common hepatic duct or common bile duct. f Three ducts with creation of one common truck for two ducts and separately anastomosis to left and right hepatic ducts

\section{Results}

\section{Overall results}

The overall incidence of biliary complications after LDLT in our series was $23.3 \%$ (in seven of 30 recipients). Median follow-up was 50 months, ranging from 36 to 69 months. The early incidence ( $\leq 90$ days) of biliary complications after LDLT was $13.3 \%$ (in four of 30 recipients), and the late incidence ( $>90$ days) of biliary complications was $10 \%$ (in three of 30 recipients).

Comparison of recipient data with or without biliary complications

There was no significant difference between recipients with or without biliary complications in terms of donor or recipient age, sex ratio, body mass index, graft weight, graft/recipient weight ratio, cold and warm ischemic time of the graft, MELD score, and etiology of recipients (Table 1). In comparison with recipients without biliary complications, the median operative time was slightly longer, without reaching statistical significance, in recipients with biliary complications. There was no difference in terms of the levels of transaminases between recipients with or without biliary complications. The cholestatic parameters were elevated on posttransplant day 7 in recipients with biliary complications as compared with those without biliary complications. Furthermore, the hospital stay was prolonged in recipients with biliary complications as compared with those without biliary complications (Table 2). Interestingly, there was no hospital mortality in patients with biliary complication. Hospital mortality was occurred in (four of 24) recipients without biliary complications. On multivariate analysis, no single risk factor associated with biliary complication could be identified.

\section{Methods for biliary reconstruction}

In the study, the primary intension for biliary reconstruction is duct-to-duct hepaticocholedochostomy. The types and frequency of the individual method used are summarized in Table 3. There was one graft hepatic duct in 19 of 30 recipients. Therefore, the most frequent method for biliary reconstruction in our series was end-to-end anastomosis between a solitary graft hepatic duct and the recipient common hepatic duct using a continuous suture technique $(n=18)$. End-to-side anastomsis was done between the solitary graft bile duct and the recipient common bile duct due to the duct lumen incongruence in one case. Complex reconstruction of the biliary tract (Fig. 1b-f) was done in grafts with biliary variances. There were more than two hepatic ducts in 11 of 30 grafts. The gap between the orifices 
Table 1 Comparison of demographic data of patients with or without biliary complication

\begin{tabular}{|c|c|c|c|c|}
\hline & \multicolumn{2}{|c|}{ With biliary $\mathrm{Cx}(n=7)$} & \multicolumn{2}{|c|}{ Without biliary $\mathrm{Cx}(n=23)$} \\
\hline \multicolumn{5}{|l|}{ Donor } \\
\hline Age & 39 & $(24-44)$ & 25 & $(20-55)$ \\
\hline $\operatorname{Sex}(F / M)$ & $1 / 6$ & & $8 / 15$ & \\
\hline BMI & 25.6 & $(21.5-28.6)$ & 24.3 & $(20.4-30.5)$ \\
\hline \multicolumn{5}{|l|}{ Recipient } \\
\hline Age & 46 & $(25-60)$ & 51 & $(16-66)$ \\
\hline $\operatorname{Sex}(\mathrm{F} / \mathrm{M})$ & $3 / 4$ & & $7 / 16$ & \\
\hline BMI & 23 & $(15.5-40.5)$ & 26.9 & $(15.6-37.2)$ \\
\hline labMELD & 13 & $(10-20)$ & 18 & $(10-36)$ \\
\hline Preoperative ICU stay & 0 & & 3 of 23 & $13 \%$ \\
\hline Renal failure & 0 & & 5 of 23 & $22 \%$ \\
\hline \multicolumn{5}{|l|}{ Etiology } \\
\hline HepB cirrhosis & 2 of 7 & & 6 of 23 & \\
\hline HepC cirrhosis & 2 of 7 & & 2 of 23 & \\
\hline Alcoholic cirrhosis & 1 of 7 & & 1 of 23 & \\
\hline Crytogenic cirrhosis & 0 of 7 & & 3 of 23 & \\
\hline $\mathrm{PBC}$ & 0 of 7 & & 1 of 23 & \\
\hline $\mathrm{HCC}$ & 1 of 7 & & 6 of 23 & \\
\hline Budd-Chiari Syn & 1 of 7 & & 2 of 23 & \\
\hline Oxalosis & 0 of 7 & & 1 of 23 & \\
\hline Autoimmune hepatitis & 0 of 7 & & 1 of 23 & \\
\hline
\end{tabular}

of two or three adjacent hepatic ducts were sutured to achieve one common orifice, which was subsequently anastomosed with common hepatic duct or common bile duct using the continuous suture technique in seven of 11 recipients. In three of 11 recipients, it was not possible to achieve one common orifice between two graft hepatic ducts; therefore, two graft hepatic ducts were anastomosed separately with the common hepatic duct and the cystic duct of recipient, respectively. In one graft with three hepatic ducts, one common orifice was achieved between two adjacent hepatic ducts of graft and was then anastomosed with the left hepatic duct of the recipient. The third graft hepatic duct was subsequently anastomosed with the right hepatic duct of recipient.

\section{Biliary complications and their managements}

The incidence of biliary complications in our series of ductto-duct hepaticocholedochostomy was $13.3 \%$ (in four of 11 recipients) in early posttransplant phase (less than 90 days) and $10 \%$ (in three of 11 recipients) in later posttransplant phase (more than 90 days, Table 3).

In early posttransplant phase, biliary stricture developed in two recipients with one graft hepatic duct and one anastomosis (Table 3, Fig. 2a). It was treated with early reoperation and Roux-en-Y hepaticojejunostomy in one recipient. This recipient recovered adequately and was discharged with normal liver biochemistry. The second patient with biliary stricture was treated via endoscopic retrograde cholangiopancreatography (ERCP), unfortunately leading to biliary perforation in the region of anastomosis and also secondary branches of the graft hepatic duct. The resulting bile leaks could not be adequately treated by placement of intraductal stents. It was subsequently treated with reoperation and Roux-en-Y hepaticojejunostomy. The recipient recovered thereafter and discharged with normal liver biochemistry. Biliary leakage occurred in two of the recipients, one with one graft hepatic duct and one anastomosis, and the other one with two graft hepatic ducts and two anastomoses. Both recipients were treated with reoperation and Roux-en-Y hepaticojejunostomy. They were discharged with normal liver biochemistry.

In later posttransplant phase, biliary stricture developed in two recipients, one with one graft hepatic duct and one anastomosis, and the other one with two graft hepatic ducts and one anastomosis (Table 3, Fig. 2b). Both recipients were treated primarily with ERCP and with placement of intraductal stent. They recovered adequately and were discharged with normal liver biochemistry. One recipient developed biliary leakage at bile duct anastomotic site leading to formation of bilioma, which resulted in extrahepatic bile duct necrosis with normal hepatic artery perfusion at 6 month after transplantation. Reoperation and reconstruction of the biliary tract with Roux-en-Y hepaticojejunostomy were performed. The patient recovered from the operation and was discharged with normal liver biochemistry. Data were summarized in Fig. 2. 
Table 2 Comparison of data of patients with or without biliary complication

\begin{tabular}{|c|c|c|c|c|c|}
\hline & & \multicolumn{2}{|c|}{ With biliary $\mathrm{Cx}(n=7)$} & \multicolumn{2}{|c|}{ Without biliary $\mathrm{Cx}(n=23)$} \\
\hline \multicolumn{6}{|l|}{ Graft status } \\
\hline Graft size (g) & & 880 & $(600-980)$ & 825 & $(620-1,400)$ \\
\hline GW/BW & & 1.36 & $(1.15-1.46)$ & 1.28 & $(0.77-2.1)$ \\
\hline Cold ischemic time (min) & & 240 & $(139-360)$ & 145 & $(71-246)$ \\
\hline Warm ischemic time (min) & & 75 & $(60-120)$ & 60 & $(21-130)$ \\
\hline \multicolumn{6}{|l|}{ Operative data } \\
\hline \multicolumn{6}{|l|}{ Donor } \\
\hline Operation time (min) & & 315 & $(235-500)$ & 300 & $(245-420)$ \\
\hline Blood loss (mL) & & 675 & $(500-950)$ & 600 & $(300-900)$ \\
\hline Intraoperative blood transfusion & & 0 & & 0 & \\
\hline Intraoperative FFP transfusion & & 0 & & 0 & \\
\hline Intraoperative platelet transfusion & & 0 & & 0 & \\
\hline \multicolumn{6}{|l|}{ Recipient } \\
\hline Operation time (minutes) & & 540 & $(248-570)$ & 448 & $(190-720)$ \\
\hline Blood loss (mL) & & 1,500 & $(800-6,250)$ & 1,200 & $(200-2,500)$ \\
\hline Intraoperative blood transfusion & & 2 & $(0-4)$ & 2 & $(0-7)$ \\
\hline Cell saver reinfusion $(\mathrm{mL})$ & & 410 & $(250-1,244)$ & 425 & $(0-750)$ \\
\hline Intraoperative FFP transfusion & & 4 & $(2-19)$ & 2 & $(0-25)$ \\
\hline Intraoperative platelet transfusion & & 0 & $(0-1)$ & 0 & $(0-1)$ \\
\hline \multicolumn{6}{|l|}{ Postoperative data } \\
\hline \multicolumn{6}{|l|}{ Donor } \\
\hline \multirow[t]{3}{*}{ Peak serum ALT } & Day 1 & 259 & $(153-336)$ & 267 & $(139-458)$ \\
\hline & Day 3 & 165 & $(130-239)$ & 150 & $(58-356)$ \\
\hline & Day 7 & 66 & $(31-92)$ & 47 & $(19-220)$ \\
\hline \multirow[t]{3}{*}{ Peak total serum bilirubin } & Day 1 & 1.9 & $(1.1-3.5)$ & 2.2 & $(1.5-6.0)$ \\
\hline & Day 3 & 3.2 & $(1.4-3.5)$ & 2.2 & $(1.0-7.6)$ \\
\hline & Day 7 & 2.1 & $(1.6-3.6)$ & 1.4 & $(0.7-4.7)$ \\
\hline Renal failure & & 0 & & 0 & \\
\hline Reoperation & & 0 & & 0 & \\
\hline ICU stay & & 2 & $(1-4)$ & 3 & $(1-5)$ \\
\hline Hospital stay & & 14 & $(12-63)$ & 10 & $(8-23)$ \\
\hline Mortality & & 0 & & 0 & \\
\hline \multicolumn{6}{|l|}{ Recipient } \\
\hline \multirow[t]{3}{*}{ Peak serum ALT } & Day 1 & 240 & $(43-1,972)$ & 223 & $(37-554)$ \\
\hline & Day 3 & 321 & $(194-2,865)$ & 223 & $(60-657)$ \\
\hline & Day 7 & 108 & $(40-485)$ & 88 & $(28-343)$ \\
\hline \multirow[t]{3}{*}{ Peak total serum bilirubin } & Day 1 & 4.2 & $(2.3-12.6)$ & 4.8 & $(0.5-26.8)$ \\
\hline & Day 3 & 4.7 & $(1.1-7.0)$ & 2.2 & $(0.3-20.9)$ \\
\hline & Day 7 & 4.3 & $(1.0-10.2)$ & 2.1 & $(0.41-28.1)$ \\
\hline Renal failure & & 0 of 7 & $0.00 \%$ & 6 of 23 & $26 \%$ \\
\hline Reoperation & & 4 of 7 & $57.10 \%$ & 6 of 23 & $26.00 \%$ \\
\hline ICU stay & & 8 & $(3-14)$ & 7 & $(1-19)$ \\
\hline Hospital stay & & 32 & $(17-39)$ & 28 & $(6-64)$ \\
\hline Mortality & & 0 & & 5 of 23 & $21.70 \%$ \\
\hline Survival (3 years) & & 7 of 7 & $100 \%$ & 18 of 23 & $78 \%$ \\
\hline
\end{tabular}

\section{Discussion}

In this cohort study, we demonstrated the methods for duct-toduct hepaticocholedochostomy with relatively low incidence of biliary complications in LDLT. There is no consensus among transplant surgeons regarding the biliary reconstruction in LDLT. This is, on one hand, due to the anatomical variance of biliary tract in right lobe graft [11]; on the other hand, the 
Table 3 Data of duct-to-duct biliary reconstruction

\begin{tabular}{|c|c|c|c|c|c|c|}
\hline \multirow[t]{2}{*}{ No. of graft bile ducts and anastomosis } & \multirow[t]{2}{*}{ No. } & \multirow[t]{2}{*}{ Methods } & \multicolumn{2}{|c|}{ Early Cx. (<90days) } & \multicolumn{2}{|c|}{ Late Cx. (>90days) } \\
\hline & & & Stricture $(\%)$ & Leakage $(\%)$ & Stricture $(\%)$ & Leakage $(\%)$ \\
\hline $1 \mathrm{duct} / 1$ anastomosis & 19 & A & 10.5 & 5.3 & 5.3 & 0 \\
\hline 2 ducts/1 anastomosis & 6 & $\mathrm{~B} / \mathrm{E}$ & 0 & 0 & 5.3 & 0 \\
\hline 3 ducts/1 anastomosis & 1 & $\mathrm{C}$ & 0 & 0 & 0 & 0 \\
\hline 2 ducts/2 anastomosis & 3 & $\mathrm{D}$ & 0 & 33.3 & 0 & 0 \\
\hline 3 ducts $/ 2$ anastomosis & 1 & $\mathrm{~F}$ & 0 & 0 & 0 & 0 \\
\hline Total & 30 & & 6.7 & 6.7 & 6.7 & 0.0 \\
\hline
\end{tabular}

methods for the biliary reconstruction may largely depend upon the experiences of transplant surgeons. The improvement of the overall results of biliary reconstruction in recent years is probably attributed by the more precise investigation of the location of biliary tract and its division to avoid two or three bile duct openings and by the modification of surgical technique in the biliary reconstruction [5]. A significant reduction of biliary complications was observed in recently reported series. However, up to $40 \%$ biliary complications are still the most serious morbidity and, in some cases, lead to hospital mortality in the recipients of LDLT [8].

For the precise investigation of graft biliary tract, it has been suggested that preoperative magnetic resonance cholangiopancreatography (MRCP) and ERCP may be helpful in understanding the secondary branches of biliary tract in right lobe graft. However, similar results can also be achieved by an intraoperative cholangiography, which may be more convenient and helpful to the transplant surgeons to make the decision [5]. Nowadays, intraoperative cholangiography has become a standard procedure in donor operation of LDLT. The need of preoperative MRCP and ERCP for donor operation is still an open question. We did not perform preoperative ERCP and MRCP in our study. The overall rate of two or three graft hepatic duct openings was $36.6 \%$ in our series, which is comparable with the recently reported series with or without preoperative ERCP and/or MRCP, ranging from $39.7 \%$ to $50 \%$ [9]. This may suggest that preoperative ERCP and MRCP are not a must for donor operation. An intraoperative cholangiography is sufficient to identify the cutting line of right hepatic duct.

Reconstruction of biliary tract with Roux-enhepaticojejunostomy was a dominant procedure in early series of LDLT [5], which showed an acceptable result in terms of biliary complications through the improvement of surgical technique. Interestingly, duct-to-duct hepaticocholedochostomy has become more popular in recent series of LDLT among transplant centers. Reasons for popularization of duct-to-duct hepaticocholedochostomy may consist of technically easier and quicker procedure, preservation of physiologic bilioenteric continuity, and easier endoscopic intervention after LDLT. Indeed, the better understanding of the blood supply of bile ducts and its anatomical variation is the basis for the improvement of surgical technique for biliary reconstruction. Therefore, the preservation of bile duct vascularization has become a critical step during donor right lobe hepatectomy [11]. Since it is possible to obtain single graft hepatic duct orifice in more than $50 \%$ of grafts, duct-to-duct hepaticocholedochostomy is more feasible than Roux-en-choledochojejunostomy.

There is no standard procedure for the duct-to-duct hepaticocholedochostomy in LDLT. In general, it is believed that a duct-to-duct hepaticocholedochostomy can always be done between two good vascularized bile ducts without extensive anastomotic tension. In our study, superior attention was paid to the preservation of axial periductal microcirculation and avoidance of the dissection of the connective tissue between right hepatic artery and right hepatic duct during donor right lobe hepatectomy. The graft hepatic ducts would be re-examined and rinsed carefully with saline in backtable. The decision on how bile ducts would be reconstructed would be made in this period. Moreover, superior attention would also be paid in preservation of sufficient length of good vascularized bile duct for the tension-free anastomosis in recipient hepatectomy. It might also be helpful by doing the anastomosis with microsurgical technique [12]. The duct-
Fig. 2 Overview of surgical techniques, management, and outcomes in seven of 30 recipients with biliary complications (1D/1A one duct with one anastomosis)

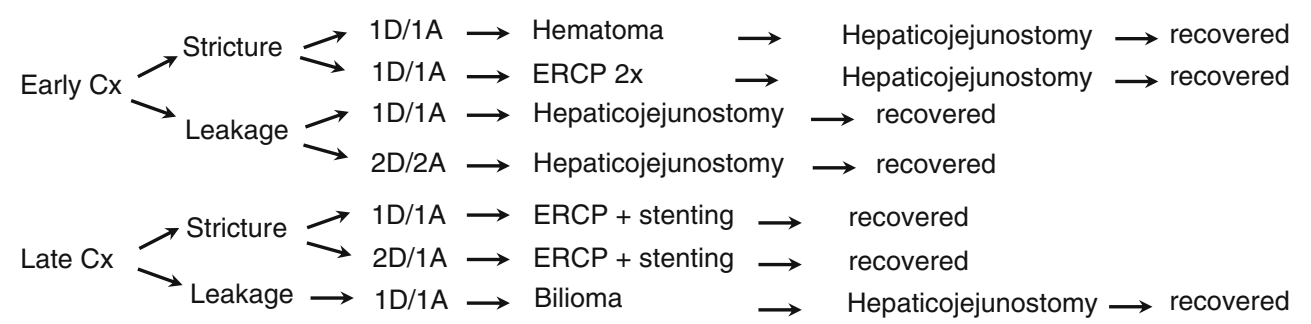


to-duct anastomosis with or without previous graft ductoplasty was done using continuous suture technique in all cases of our series (see Fig. 1). With this strategy, there were $13.3 \%$ biliary complications in early posttransplant phase and $10 \%$ in later posttransplant phase in our series, which is lower than the recent reported series. In addition, there was no mortality that was associated with biliary complications in our series of LDLT.

There was still the discussion among transplant surgeons, whether intraductal stent or T-tube should be placed during or after duct-to-duct hepaticocholedochostomy [5, 13, 14]. In our study, no intraductal stent was placed during the biliary reconstruction. T-tube would generally be placed in highrisked duct-to-duct hepaticocholedochostomy. T-tube was placed in four of seven recipients with graft ductoplasty. There were not biliary complications in recipients with Tdrainage, whereas one of three recipients with graft ductoplasty, who did not have T-drainage, suffered from biliary stricture in later posttransplant phase (data not shown). This suggests that T-drainage may be helpful after the "highrisked" duct-to duct anastomosis in patients with more than two ducts anastomosis and under perioperative high-dose of catecholamine or under hemofiltration. However, this question can only be answered with a prospective study.

Despite the technical refinement, biliary complications are still unavoidable in most of transplant centers. The management of biliary complications is a main issue in the control of posttransplant morbidity of LDLT recipients. Unlike the reported series, we preferred to solve the early biliary stricture or leakage with reoperation and with Rouxen-hepaticojejunostomy, once indicated. There was iatrogenic bile duct perforation occurring in one case of our series with biliary stricture in early posttransplant phase, which was treated with endoscopic intervention. This may indicate that there is a higher risk of iatrogenic damage of graft bile tract in early posttransplant phase. Reoperation might superior than endoscopic intervention, whenever the recipient's condition allows in this period. To treat the recipients with late biliary complications, which are dominated with biliary stricture, endoscopic intervention is one of the best choices. In our study, two recipients with biliary stricture were successfully treated with ERCP and stenting.

In conclusion, duct-to-duct hepaticocholedochostomy with continuous suture technique is a feasible and safe procedure for the biliary reconstruction in LDLT. This was reflected by low incidence of biliary complications in our series. Once the complications occur, aggressive approach with reoperation is recommended in early posttransplant phase. Endoscopic intervention can be preserved for the late biliary complication.

Authors' contributions TYT: study design, acquisition, analysis and interpretation of data, wrote the manuscript, revision of manuscript.

HJS: study design and conception, revision of manuscript.

AO: study design and conception, revision of manuscript.

\section{Conflicts of interest None.}

Open Access This article is distributed under the terms of the Creative Commons Attribution Noncommercial License which permits any noncommercial use, distribution, and reproduction in any medium, provided the original author(s) and source are credited.

\section{References}

1. Fan ST (2006) Live donor liver transplantation in adults. Transplantation 82:723-732

2. Lo CM, Fan ST, Liu CL, Yong BH, Wong Y, Lau GK, Lai CL et al (2004) Lessons learned from one hundred right lobe living donor liver transplants. Ann Surg 240:151-158

3. Fan ST, Lo CM, Liu CL (2000) Technical refinement in adult-toadult living donor liver transplantation using right lobe graft. Ann Surg 231(1):126-131

4. Ishiko T, Egawa H, Kasahara M, Nakamura T, Oike F, Kaihara S, Kiuchi T, Uemoto S, Inomata Y, Tanaka K (2002) Duct-to-duct biliary reconstruction in living donor liver transplantation utilizing right lobe graft. Ann Surg 236:235-240

5. Fan ST, Lo CM, Liu CL, Tso WK, Wong J (2002) Biliary reconstruction and complications of right lobe live donor liver transplantation. Ann Surg 236:676-683

6. Grewal HP, Shokouh-Amiri MH, Vera S, Stratta R, Bagous W, Gaber AO (2001) Surgical technique for right lobe adult living donor liver transplantation without venovenous bypass or portocaval shunting and with duct-to-duct biliary reconstruction. Ann Surg 233(4):502-508

7. Malago M, Testa G, Hertl M, Lang H, Andreas P, Frilling A, Treichel U, Broelsch C (2002) Biliary reconstruction following right adult living donor liver transplantation end-to-end or endto-side duct-to-duct anastomosis. Langenbeck's Arch Surg 387:37-44

8. Tashiro H, Itamoto T, Sasaki T, Ohdan H, Fudaba Y, Amano H, Fukuda S, Nakahara H, Ishiyama K, Ohshita A, Kohashi T, Mitsuta H, Chayama K, Asahara T (2007) Biliary complications after duct-toduct biliary reconstruction in living-donor liver transplantation: causes and treatment. World J Surg 31(11):2222-2229

9. Kasahara M, Egawa H, Takada Y, Oike F, Sakamoto S, Kiuchi T, Yazumi S, Shibata T, Tanaka K (2006) Biliary reconstruction in right lobe living-donor liver transplantation: comparison of different techniques in 321 recipients. Ann Surg 243(4):559-566

10. Kohler S, Pascher A, Mittler J, Neumann U, Neuhaus P, Pratschke J (2009) Management of biliary complications following living donor liver transplantation - a single center experience. Langenbeck's Arch Surg 394(6):1025-1031, Epub 2009 May 27

11. Ohkubo M, Nagino M, Kamiya J, Yuasa N, Oda K, Arai T, Nishio H, Nimura Y (2004) Surgical anatomy of the bile ducts at the hepatic hilum as applied to living donor liver transplantation. Ann Surg 239(1):82-86

12. Lin TS, Concejero AM, Chen CL, Chiang YC, Wang CC, Wang SH, Liu YW, Yang CH, Yong CC, Jawan B, Cheng YF (2009) Routine microsurgical biliary reconstruction decreases early anastomotic complications in living donor liver transplantation. Liver Transplant 15(12):1766-1775

13. Liu CL, Lo CM, Chan SC, Fan ST (2004) Safety of duct-to-duct biliary reconstruction in right-lobe live-donor liver transplantation without biliary drainage. Transplantation 77(5):726-732

14. Hashimoto M, Sugawara Y, Tamura S, Kishi Y, Matsui Y, Kaneko J, Makuuchi M (2006) T-tube drainage for biliary stenosis after living donor liver transplantation. Transplantation 81(2):293-295 